\title{
Manipulation of the ATP pool as a tool for metabolic engineering
}

Oliver Hädicke*, Steffen Klamt

Max Planck Institute for Dynamics of Complex Technical Systems

Sandtorstrasse 1, Magdeburg, 39106, Germany

* To whom correspondence should be addressed:

Oliver Hädicke

Max Planck Institute for Dynamics of Complex Technical Systems, Germany

Sandtorstrasse 1

D-39106 Magdeburg, Germany

Phone: ++49 3916110489

Email: haedicke@mpi-magdeburg.mpg.de 


\section{Abstract}

Cofactor engineering has been long identified as a valuable tool for metabolic engineering. Besides interventions targeting the pools of redox cofactors, many studies addressed the adenosine pools of microorganisms. In this minireview we discuss interventions that manipulate the availability of ATP with a special focus on ATP wasting strategies. We analyse the importance to fine-tune the ATP yield along a production pathway to balance process performance parameters like product yield and volumetric productivity.

Key words: metabolic engineering, ATP futile cycling, product yield, volumetric productivity

\section{Introduction}

The shift from petrochemical to biotechnological synthesis of desired chemicals and biofuels is one major goal for the development of sustainable production processes. Such a shift can only be successful if the designed processes are not only advantageous with respect to environmental issues but at least as economically viable as their petrochemical counterparts [1, 2]. Key parameters that determine the quality of production processes with respect to economical needs are product yield, product titer, and productivity [3, 4].

Metabolic engineering as one enabling technology within this field aims at the optimization of intracellular processes of microorganisms to improve these key parameters [5]. Manipulation of the cofactor pools has been long identified as a valuable intervention target [6-8]. Direct effects, like increasing the reducing power for the production of relatively reduced products, as well as indirect effects such as 
obligatory coupling of product excretion and biomass synthesis can be enforced via cofactor manipulation [7-9].

Two of the most important cofactors that are accessible for interventions are the $\mathrm{NAD}(\mathrm{P}) \mathrm{H}$ and the ATP pool. Interventions targeting the $\mathrm{NAD}(\mathrm{P}) \mathrm{H}$ pool often ensure that product excretion is the only possibility to regenerate the redox cofactors $N A D(P)$. This approach is especially appropriate for relatively reduced products like organic acids, alcohols, or terpenoids. Successful examples are given by microbial production of succinate [10] or 1,3-propanediol [11]. An increased supply of reducing power can further increase the beneficial effects of these interventions [12].

Here, we review engineering strategies targeting the ATP pool with a special focus on interventions that imply elevated "ATP wasting", e.g. through ATP futile cycling or implementation of pathways with low ATP yield. We discuss results of experimental studies with interventions targeting the ATP pool for Escherichia coli and Saccharomyces cerevisiae with respect to process performance. Further, the general relevance of the ATP pool as engineering target is analysed and finally inherent trade-offs between important process performance parameters (e.g. yield and specific productivity versus volumetric productivity) when employing ATP wasting strategies are discussed.

\section{Experimental studies manipulating the ATP pool in Escherichia coli}

Interventions related to ATP synthesis (generated along metabolic pathways with a positive net ATP yield) either increase or decrease the amount of ATP that is available for biomass synthesis as well as maintenance and other processes in the cell. ATP conserving manipulations are especially appropriate for products whose 
syntheses are ATP neutral or even demand the additional investment of ATP. These manipulations include, for example, the overexpression of thermodynamically favorable carboxylation reactions like phosphoenolpyruvate carboxykinase (pck) (compared to pyruvate carboxylase) [13, 14]. These interventions have been successfully applied for construction of strains producing succinate [10, 15], or recombinant proteins [13]. Besides increased product yields, higher growth rates were observed for the respective producer strains.

If product synthesis is accompanied by ATP generation, interventions decreasing the availability of ATP have also been shown to imply beneficial effects on process parameters [16-18]. Expression of the $F(1)$ part of the membrane-bound $(F(1) F(0))$ $\mathrm{H}(+)$-ATP synthase constantly consuming ATP without pumping protons has been shown to increase the substrate uptake and glycolytic flux and decrease the biomass production at aerobic conditions $[18,19]$. The excretion rate of fermentation products could also be increased by implementing ATP futile cycles aerobically [17, 20, 21]. For anaerobic conditions, only recently an elevated specific lactate production rate was observed by implementing an ATP futile cycle between pyruvate and PEP through overexpression of the phosphoenolypyruvate synthase [22]. In concordance with other experimental results, this study also reported a decreased growth rate and significantly increased glucose uptake rate.

\section{Experimental studies manipulating the ATP yield in Saccharomyces cerevisiae}

Experimental results of ATP targeting interventions in $S$. cerevisiae have been reviewed in detail elsewhere [6]. Three engineering targets of suitable interventions to modulate the ATP yield were identified and discussed thoroughly: i) uptake and phosphorylation of sugars, ii) stoichiometries of oxidative phosphorylation and $\mathrm{H}^{+}$- 
ATPase, iii) C3 $\rightarrow$ C4 carboxylation reactions. Here, we briefly discuss one exemplary study for each class and discuss the results with respect to growth and product yields.

One approach to engineer the uptake and phosphorylation systems of sugars is the replacement of facilitated diffusion systems by proton symporters or, alternatively, by ATP-driven transporters thereby lowering the excess ATP production [23]. Using this engineering target and a combination of metabolic and evolutionary engineering resulted in an ethanol production strain with a $30 \%$ decreased biomass yield and a $11 \%$ increased ethanol yield relative to a reference strain [23].

To reduce the $\mathrm{P} / \mathrm{O}$ ratio of respiration in $S$. cerevisiae two different metabolic engineering strategies have been described. Either alternative oxidases that transfer electrons from cytochrome $c$ to molecular oxygen without proton translocation [24] or bacterial water-forming NADH oxidases that bypass the electron transport chain can be expressed. Both approaches led to decreased aerobic biomass yields and shifted the product profile to more oxidized metabolites (due to more oxidized NADH pools) [25-28].

For the production of dicarboxylic acids one major engineering goal is to increase the ATP yield of product formation since the optimal synthesis pathways have only low or even zero ATP yields (depending on the extracellular conditions). Free-energy (ATP) conservation can be realized via interventions at several $\mathrm{C} 3 \rightarrow \mathrm{C} 4$ carboxylation reactions $[14,29]$. For example, using either malic enzyme or PEP carboxykinase instead of pyruvate carboxylase increases the overall ATP yield by one ATP per C3 $\rightarrow$ C4 carboxylation event. The reported activity of these replacements is in the opposite of the typical decarboxylating direction, enables anaerobic malic acid 
production and increases the ATP yield for redox-neutral succinic acid production significantly [15, 30-33].

Besides these three engineering targets, the extracellular conditions play an important role for the overall ATP yield of product synthesis pathways [34, 35]. For example, export of dicarboxylic acids under industrially desirable conditions (high titer and low $\mathrm{pH}$ ) requires the investment of free energy and thereby influences the overall ATP yield via product synthesis. The applied experimental approaches for manipulating the ATP yield of the discussed studies and their major implications on the designed strains are summarized in Table 1.

\section{ATP wasting as general engineering target}

Excess ATP generated via product pathways can be used by microorganisms for growth, maintenance, or other non-product related pathways. The reciprocal relationship between growth yield and product yield (the more substrate is used for biomass generation the less is available for product synthesis) makes the ATP pool a highly relevant target for the design of metabolic engineering strategies aiming at high product yields. If product synthesis is accompanied by ATP generation, it is desirable to design product synthesis pathways with low but positive ATP yields such that the host cells can generate only just as much free energy as needed for growth (the growth rate can get reduced compared to a reference strain).

\section{Effects of ATP wasting on process parameters}

Optimal product yields can be achieved if the product synthesis pathways are the only ATP source since a higher fraction of the substrate must be directed to the 
product to generate the ATP needed for biomass synthesis. Therefore, a primary effect of an ATP wasting intervention is the increase of product yield (Figure 1). Since the specific productivity only depends on the product yield and substrate uptake rate, this process parameter is increased by ATP wasting interventions (Figure 1). Likewise, a second primary effect of ATP wasting is the reduction of the biomass yield implying reduced specific growth rates (as long as the substrate uptake rate is not increased; see below).

Further implications on process parameters can be concluded through secondary interdependencies. The reduced specific growth rate implies, at a specific time point during the process, a reduced volumetric growth rate and eventually a reduced biomass concentration (compared to a process without ATP wasting). Therefore, the volumetric substrate uptake rate and the volumetric productivity are negatively affected. The same holds for the concentrations of biomass and product (titer) for time points before the substrate is completely consumed.

However, a third primary effect of ATP wasting that was observed experimentally (but cannot be proven as a general consequence of ATP wasting) is the increase of the specific substrate uptake rate. This effect alleviates the negative implications on volumetric growth rate and productivity. Theoretically, the cell could counteract the loss of ATP completely by increasing the substrate uptake rate by the amount required to synthesize the lost ATP (so that the growth rate is not impaired). In such a (unlikely) scenario, in addition to the product yield and specific productivity, even the volumetric productivity could be increased. 


\section{Effects of ATP wasting on volumetric productivity with constant substrate uptake rate}

Assuming that i) ATP wasting has no effect on the substrate uptake rate and ii) the amount of substrate is limited, concrete effects of ATP wasting on process parameters can be concluded (Figure 2). The exact nonlinear relationship between the analysed process parameters depends on concrete parameter values. However, qualitatively The functional relationship qualitatively follows an inverse quadratic shape. A detailed mathematical model is given by [3]

However, the particular effect of ATP wasting interventions on the volumetric productivity depends on the characteristics of the reference strain $\mathrm{S}_{0}$. Assume that the intracellular flux distribution of $S_{0}$ corresponds roughly to the situation displayed left in Figure 2. A first decrease of the ATP amount available for growth (and other processes) will shift the flux distribution close to a balanced biomass and product synthesis (displayed in the centre of Figure 2) and thus an increased productivity. The influence of interventions targeted to increase the availability of ATP can be discussed analogously. Since increased amounts of ATP lead to decreased product yields but elevated growth rates, the intracellular flux distributions will be shifted left in Figure 2. Consequently, the volumetric productivity is decreased if $S_{0}$ shows a general flux distribution displayed in the centre or increased if the flux distribution of $\mathrm{S}_{0}$ rather resembles the situation displayed right in Figure 2.

These analyses show that there exists an inherent trade-off between high product yield (in $\mathrm{mol} / \mathrm{mol}$ ) and specific productivity (in $\mathrm{mmol} / \mathrm{gram}$ dry biomass weight $/ \mathrm{h}$ ) on the one hand and a high volumetric productivity (in $\mathrm{mmol} / / \mathrm{h}$ ) on the other hand [3]. The first two parameters are optimal with small biomass yield and growth rates whereas the later requires large amounts of biomass and thus high growth rates. 
Therefore, the volumetric productivity of strains that are designed for optimal product yields and specific productivities can be outperformed by strains with suboptimal product yields but superior growth rates. For batch fermentations, this effect increases with fermentation time as the volumetric productivity is governed by the biomass concentration of the production strain. A detailed mathematical analysis of the interdependencies of various process parameters with the given constraints is given elsewhere [3].

\section{In vitro and in vivo methods for increasing the volumetric productivity}

Experimentally, the volumetric productivity of batch processes can be increased in different ways. One approach is the application of a two-stage (dual-phase) process [36-38] to decouple the growth and production phase. For example, in the first phase, the cells can be cultivated aerobically to grow as fast as possible. Subsequently, cultivation conditions can be shifted to anaerobic production conditions with reduced growth rates.

Another approach that allows for single-phase processes, is the use of tunable promoters [39]. These promoters allow for the adjustment of the strength of ATPrelated interventions such that the trade-off between yield and productivity can be fine-tuned as desired by the user.

The implementation of genetic toggle switches enables the design of dynamic on-off regulators and optimal time points for activating the desired manipulations can be computationally determined [36]. Different experimental approaches for dynamic control of gene expression and their application to design dynamic control strategies have been reviewed recently [40]. The successful implementation of dynamic control 
of gene expression holds great potential to increase the productivity of biotechnological processes.

\section{In silico methods to identify targets for increasing the volumetric productivity}

In the last decade, a variety of computational methods have been developed to design engineering strategies that lead to increased product yields [41, 42]. However, these methods cannot be used to optimize the volumetric productivity of the overall process since their scope is limited to the intracellular flux distribution of the applied microorganism whereas the volumetric productivity relies on both, the product yield and the total biomass concentration. Recently, Zhuang et al. (2010) proposed a computational method that considered the volumetric productivity as an explicit objective [3]. However, this method assumes a fixed substrate uptake rate and needs therefore to be adapted if ATP wasting induces increased substrate uptake rates.

\section{Summary}

Examples of pathways with optimal process economy (with respect to product yield and production rate) involve fermentative, anaerobic pathways with a low, but positive ATP yield such as ethanol production by $S$. cerevisiae or lactate production by lactic acid bacteria. However, several inherent trade-offs between important process performance parameters have to be simultaneously considered when designing production strains using ATP wasting strategies. For batch fermentations these trade-offs exist between i) growth rate and product yield, ii) growth rate and productivity, iii) growth rate and titer and iv) product yield and productivity. Interventions based on ATP wasting may not only increase the product to biomass 
ratio but also the substrate uptake flux and thereby potentially even the volumetric productivity. Enforced ATP wasting as a design principle for metabolic engineering has great potential to optimize a variety of production processes if the product of interest leads to net ATP synthesis.

\section{Funding}

This work was in parts supported by the German Federal Ministry of Education and Research Biotechnologie 2020+ project CASCOO (FKZ: 031A180B)). 
Table 1 Experimental studies with manipulations of the ATP pool and resulting major implications

\begin{tabular}{lllll}
\hline Organism & Condition & Manipulation & Major implications & \\
\hline E. coli & aerobic & Enhanced ATP hydrolysis & Increased glycolytic flux, decreased growth rate & Reference \\
E. coli & aerobic & ATP futile cycling & Reduced growth rate, increased fermentation rates \\
E. coli & aerobic & ATP conservation & Increased biomass and product (succinate) yield & [18] \\
E. coli & anaerobic & ATP futile cycling & Reduced growth rate, increased lactate yield and rate & [22] \\
S. cerevisiae & anaerobic & Enforced proton symporter & Increased product yield, reduced biomass yield \\
S. cerevisiae & microaerobic & Reduced P/O ratio & Reduced biomass yield & [23] \\
S. cerevisiae & anaerobic & ATP conservation & Enables (otherwise infeasible) growth and product synthesis & [31]
\end{tabular}




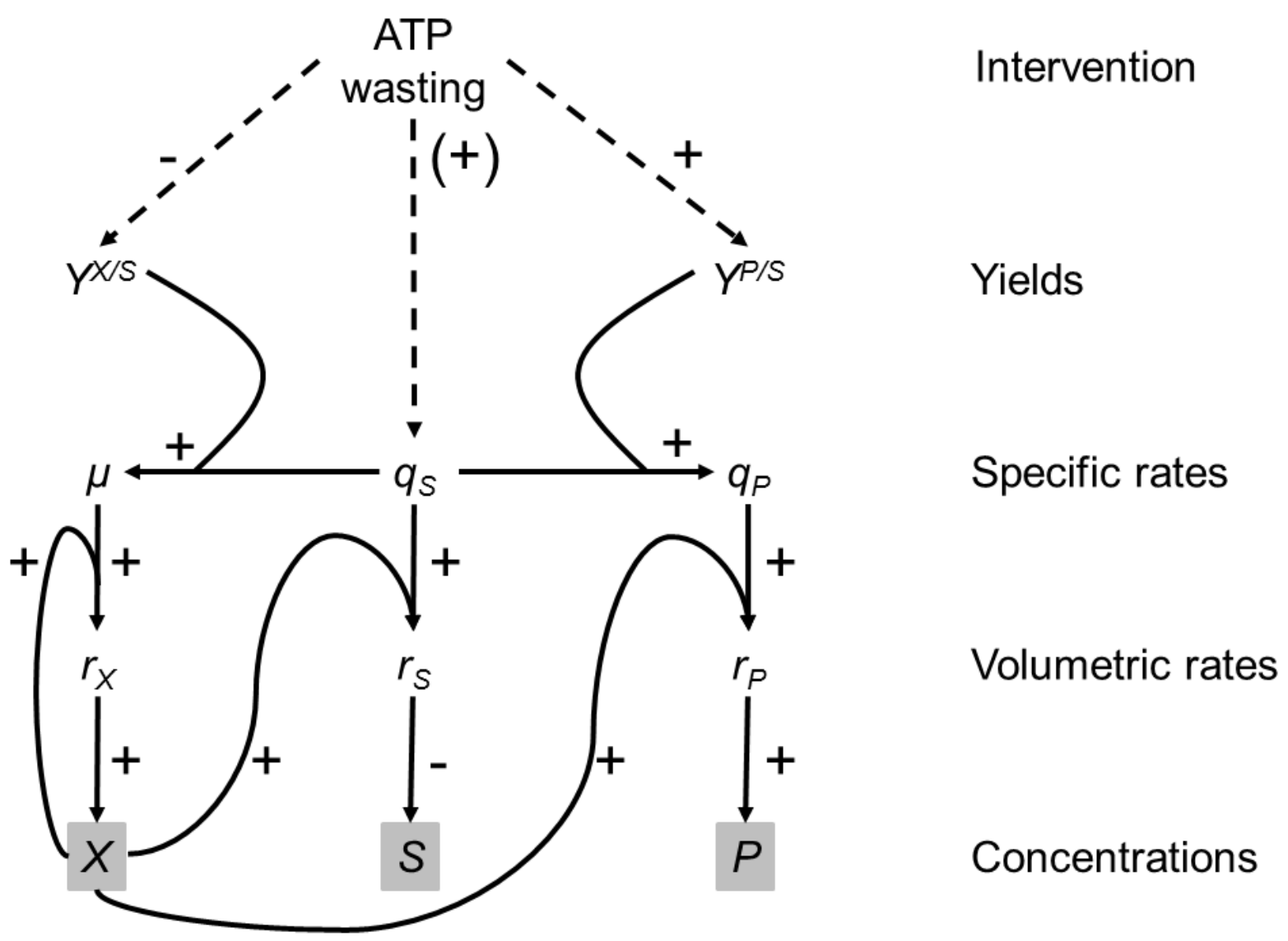

Figure 1. Primary effects of ATP wasting on performance parameters of fermentation processes. $Y^{X / S}$ biomass yield per substrate, $Y^{P / S}$ product yield per substrate, $\mu$ specific growth rate, $q_{S}$ specific substrate uptake rate, $q_{P}$ specific product excretion rate. $r_{X}, r_{S}$ and $r_{P}$ as corresponding volumetric rates. $X, S$ and $P$ as biomass, substrate and product concentrations. 


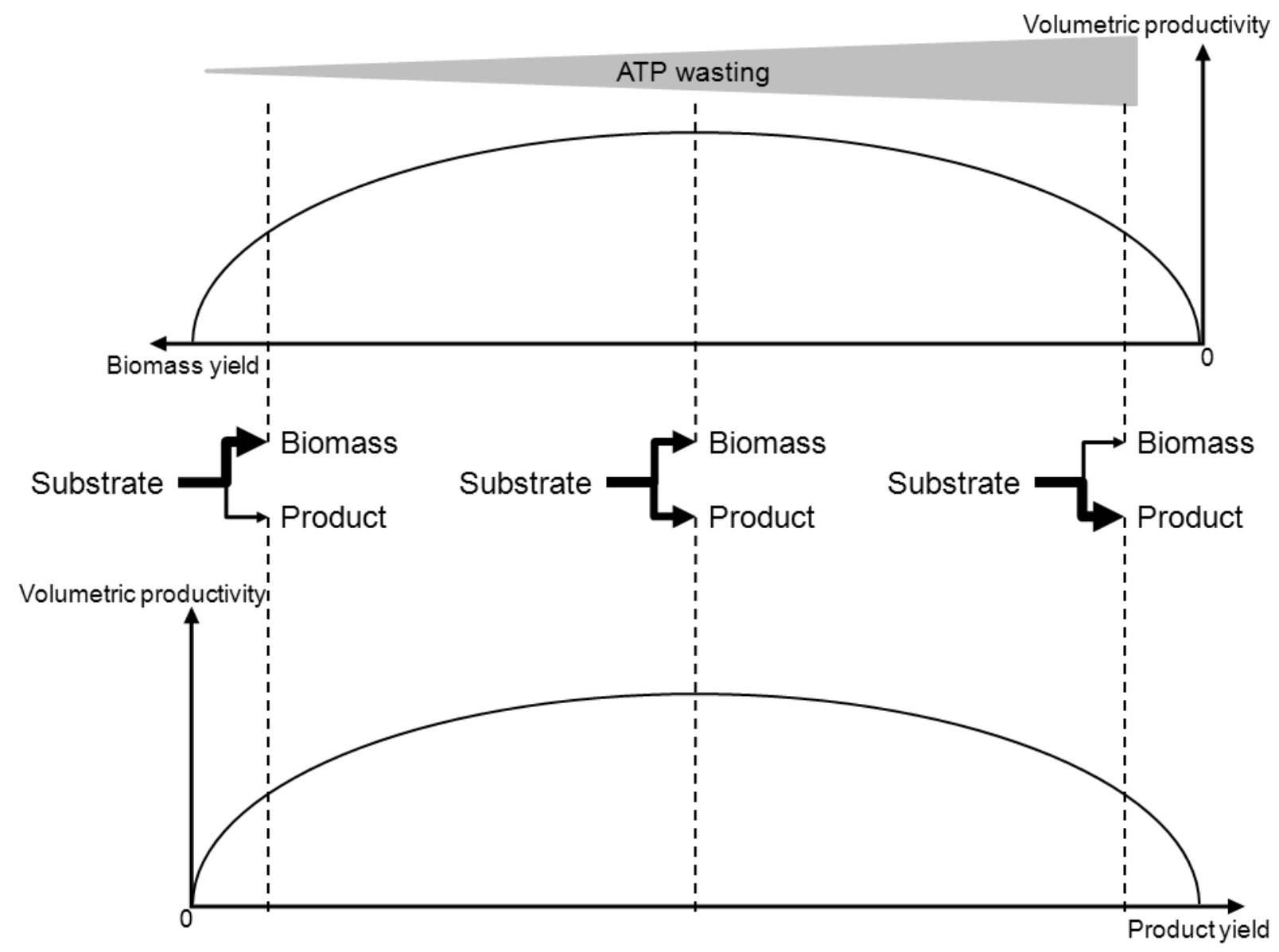

Figure 2. Relationships between specific productivity, flux partitioning (biomass vs. product), product yield, growth rate and volumetric productivity for an assumed constant substrate uptake rate 
1 Cho, C., Choi, S. Y., Luo, Z. W. and Lee, S. Y. (2014) Recent advances in microbial production of fuels and chemicals using tools and strategies of systems metabolic engineering. Biotechnology advances

2 Otero, J. M. and Nielsen, J. (2010) Industrial systems biology. Biotechnology and bioengineering. 105, 439-460

3 Zhuang, K., Yang, L., Cluett, W. R. and Mahadevan, R. (2013) Dynamic strain scanning optimization: an efficient strain design strategy for balanced yield, titer, and productivity. DySSCO strategy for strain design. BMC biotechnology. 13, 8 $4 \quad$ Park, J. H., Lee, S. Y., Kim, T. Y. and Kim, H. U. (2008) Application of systems biology for bioprocess development. Trends in biotechnology. 26, 404-412

5 Lee, J. W., Na, D., Park, J. M., Lee, J., Choi, S. and Lee, S. Y. (2012) Systems metabolic engineering of microorganisms for natural and non-natural chemicals. Nature chemical biology. 8, 536-546

6 de Kok, S., Kozak, B. U., Pronk, J. T. and van Maris, A. J. (2012) Energy coupling in Saccharomyces cerevisiae: selected opportunities for metabolic engineering. FEMS yeast research. 12, 387-397

$7 \quad$ Holm, A. K., Blank, L. M., Oldiges, M., Schmid, A., Solem, C., Jensen, P. R. and Vemuri, G. N. (2010) Metabolic and transcriptional response to cofactor perturbations in Escherichia coli. The Journal of biological chemistry. 285, 17498-17506 8 San, K. Y., Bennett, G. N., Berrios-Rivera, S. J., Vadali, R. V., Yang, Y. T., Horton, E., Rudolph, F. B., Sariyar, B. and Blackwood, K. (2002) Metabolic engineering through cofactor manipulation and its effects on metabolic flux redistribution in Escherichia coli. Metabolic engineering. 4, 182-192

9 Feist, A. M., Zielinski, D. C., Orth, J. D., Schellenberger, J., Herrgard, M. J. and Palsson, B. O. (2010) Model-driven evaluation of the production potential for growth-coupled products of Escherichia coli. Metabolic engineering. 12, 173-186 10 Singh, A., Cher Soh, K., Hatzimanikatis, V. and Gill, R. T. (2011) Manipulating redox and ATP balancing for improved production of succinate in E. coli. Metabolic engineering. 13, 76-81

11 Yu, C., Cao, Y., Zou, H. and Xian, M. (2011) Metabolic engineering of Escherichia coli for biotechnological production of high-value organic acids and alcohols. Appl Microbiol Biotechnol. 89, 573-583

12 Chemler, J. A., Fowler, Z. L., McHugh, K. P. and Koffas, M. A. G. (2010) Improving NADPH availability for natural product biosynthesis in Escherichia coli by metabolic engineering. Metabolic engineering. 12, 96-104

13 Kim, H. J., Kwon, Y. D., Lee, S. Y. and Kim, P. (2012) An engineered Escherichia coli having a high intracellular level of ATP and enhanced recombinant protein production. Appl Microbiol Biot. 94, 1079-1086

14 Kim, P., Laivenieks, M., Vieille, C. and Zeikus, J. G. (2004) Effect of overexpression of Actinobacillus succinogenes phosphoenolpyruvate carboxykinase on succinate production in Escherichia coli. Appl Environ Microbiol. 70, 1238-1241

15 Zhang, X., Jantama, K., Moore, J. C., Jarboe, L. R., Shanmugam, K. T. and Ingram, L. O. (2009) Metabolic evolution of energy-conserving pathways for succinate production in Escherichia coli. Proceedings of the National Academy of Sciences of the United States of America. 106, 20180-20185

16 Causey, T. B., Zhou, S., Shanmugam, K. T. and Ingram, L. O. (2003) Engineering the metabolism of Escherichia coli W3110 for the conversion of sugar to redox-neutral and oxidized products: homoacetate production. Proceedings of the National Academy of Sciences of the United States of America. 100, 825-832

17 Chao, Y. P., Patnaik, R., Roof, W. D., Young, R. F. and Liao, J. C. (1993) Control of gluconeogenic growth by pps and pck in Escherichia coli. Journal of bacteriology. 175, 6939-6944

18 Koebmann, B. J., Westerhoff, H. V., Snoep, J. L., Nilsson, D. and Jensen, P. R. (2002) The glycolytic flux in

Escherichia coli is controlled by the demand for ATP. Journal of bacteriology. 184, 3909-3916

19 Koebmann, B. J., Westerhoff, H. V., Snoep, J. L., Solem, C., Pedersen, M. B., Nilsson, D., Michelsen, O. and Jensen, P. R. (2002) The extent to which ATP demand controls the glycolytic flux depends strongly on the organism and conditions for growth. Molecular biology reports. 29, 41-45

20 Chao, Y. P. and Liao, J. C. (1994) Metabolic responses to substrate futile cycling in Escherichia coli. The Journal of biological chemistry. 269, 5122-5126

21 Patnaik, R., Roof, W. D., Young, R. F. and Liao, J. C. (1992) Stimulation of glucose catabolism in Escherichia coli by a potential futile cycle. Journal of bacteriology. 174, 7527-7532

22 Hädicke, O., Bettenbrock, K. and Klamt, S. (2015) Enforced ATP futile cycling increases specific productivity and yield of anaerobic lactate production in Escherichia coli. Biotechnology and bioengineering

23 Basso, T. O., de Kok, S., Dario, M., do Espirito-Santo, J. C., Muller, G., Schlolg, P. S., Silva, C. P., Tonso, A., Daran, J. M., Gombert, A. K., van Maris, A. J., Pronk, J. T. and Stambuk, B. U. (2011) Engineering topology and kinetics of sucrose metabolism in Saccharomyces cerevisiae for improved ethanol yield. Metabolic engineering. 13, 694-703

24 Joseph-Horne, T., Hollomon, D. W. and Wood, P. M. (2001) Fungal respiration: a fusion of standard and alternative components. Biochimica et biophysica acta. 1504, 179-195

25 Huh, W. K. and Kang, S. O. (1999) Molecular cloning and functional expression of alternative oxidase from Candida albicans. Journal of bacteriology. 181, 4098-4102

26 Heux, S., Cachon, R. and Dequin, S. (2006) Cofactor engineering in Saccharomyces cerevisiae: Expression of a H2Oforming NADH oxidase and impact on redox metabolism. Metabolic engineering. 8, 303-314 
Vemuri, G. N., Eiteman, M. A., McEwen, J. E., Olsson, L. and Nielsen, J. (2007) Increasing NADH oxidation reduces overflow metabolism in Saccharomyces cerevisiae. Proceedings of the National Academy of Sciences of the United States of America. 104, 2402-2407

28 Hou, J., Lages, N. F., Oldiges, M. and Vemuri, G. N. (2009) Metabolic impact of redox cofactor perturbations in Saccharomyces cerevisiae. Metabolic engineering. 11, 253-261

29 Stols, L. and Donnelly, M. I. (1997) Production of succinic acid through overexpression of NAD(+)-dependent malic enzyme in an Escherichia coli mutant. Appl Environ Microbiol. 63, 2695-2701

30 Zhang, X., Wang, X., Shanmugam, K. T. and Ingram, L. O. (2011) L-malate production by metabolically engineered Escherichia coli. Appl Environ Microbiol. 77, 427-434

31 Zelle, R. M., de Hulster, E., Kloezen, W., Pronk, J. T. and van Maris, A. J. (2010) Key process conditions for production of $C(4)$ dicarboxylic acids in bioreactor batch cultures of an engineered Saccharomyces cerevisiae strain. Appl Environ Microbiol. 76, 744-750

32 Zelle, R. M., Harrison, J. C., Pronk, J. T. and van Maris, A. J. (2011) Anaplerotic role for cytosolic malic enzyme in engineered Saccharomyces cerevisiae strains. Appl Environ Microbiol. 77, 732-738

33 Zelle, R. M., Trueheart, J., Harrison, J. C., Pronk, J. T. and van Maris, A. J. (2010) Phosphoenolpyruvate carboxykinase as the sole anaplerotic enzyme in Saccharomyces cerevisiae. Appl Environ Microbiol. 76, 5383-5389 34 van Maris, A. J., Konings, W. N., van Dijken, J. P. and Pronk, J. T. (2004) Microbial export of lactic and 3hydroxypropanoic acid: implications for industrial fermentation processes. Metabolic engineering. 6, 245-255

35 Abbott, D. A., Zelle, R. M., Pronk, J. T. and van Maris, A. J. (2009) Metabolic engineering of Saccharomyces cerevisiae for production of carboxylic acids: current status and challenges. FEMS yeast research. 9, 1123-1136 36 Anesiadis, N., Cluett, W. R. and Mahadevan, R. (2008) Dynamic metabolic engineering for increasing bioprocess productivity. Metabolic engineering. 10, 255-266

37 Gadkar, K. G., Doyle lii, F. J., Edwards, J. S. and Mahadevan, R. (2005) Estimating optimal profiles of genetic alterations using constraint-based models. Biotechnology and bioengineering. 89, 243-251

38 Soma, Y., Tsuruno, K., Wada, M., Yokota, A. and Hanai, T. (2014) Metabolic flux redirection from a central metabolic pathway toward a synthetic pathway using a metabolic toggle switch. Metabolic engineering. 23, 175-184 39 Mijakovic, I., Petranovic, D. and Jensen, P. R. (2005) Tunable promoters in systems biology. Current opinion in biotechnology. 16, 329-335

40 Venayak, N., Anesiadis, N., Cluett, W. R. and Mahadevan, R. (2015) Engineering metabolism through dynamic control. Current opinion in biotechnology. 34C, 142-152

41 Long, M. R., Ong, W. K. and Reed, J. L. (2015) Computational methods in metabolic engineering for strain design. Current opinion in biotechnology. 34C, 135-141

42 Lewis, N. E., Nagarajan, H. and Palsson, B. O. (2012) Constraining the metabolic genotype-phenotype relationship using a phylogeny of in silico methods. Nat Rev Microbiol. 10, 291-305 